\title{
A SECÇÃO DE MICOLOGIA DO DEPARTAMEN- TO DE MICROBIOLOGIA E IMUNOLOGIA
}

\author{
DR. FLORIANO DE ALMEIDA \\ (1.0 assistente $\in$ ' Docente livre de Microbiologia da Faculdade de Medicina da \\ Universidade de São Paulo)
}

Em 1926 quando ingressamos no Departamento de Microbiologia, fomos pélo prof. Souza Campos, seu Diretor, encarregados de estudar o problema da blastomicose brasileira.

$\mathrm{Na}$ mesma época por comum acordo entre os profs. Lauro Travassos è Flavio Fonseca respectivamente na regência das cadeiras de Parasitologia e de Microbiologia, a micologia que sempre fôra lecionada na primeira passou para a segunda. Mas foi sómente anos depois que os respectivos catedráticos profs. Samuel Pessôa e Souza Campos ratificaram a combinação anterior. Tendo sido desde o início encarregados da parte de micologia, procurámos angariar os elementos necessários, para organisação de uma micoteca médica. Para formação do núcleo inicial de culturas muito contribuiu a Secção de Micologia do Instituto Oswaldo Cruz, de onde trouxemos numerosas culturas. Realisando uma pesquisa cuidadosa em vários laboratórios encontramos interessante material de micoses ao mesmo tempo que obtinhamos dados estatísticos sobre as mesmas. Os casos de micoses que surgiram daí por deante permitirám-nos obter muitas culturas, assim, a micoteca que em 1931 contava apenas 200 culturas tem hoje cerca de 1.200. Da meșma maneira o material histológico proveniente de. biópsias e autópsias de casos humanos e animais foi aos poucos se acumulando de modo a termos atualmente material de mais de 300 casos nacionais e estrangeiros.

Êsse material histológico é conservado incluido em blócos de parafina porém às vêzes conservamos uma parte em líquido fixador, mórmente quando é uma peça grande que é então colocada em vidros especiais para a formação de um pequeno museu, que conta já com várias peças. Dêsse material foram feitas preparações histológicas que servem para pesquisas e tambem para o ensino prático das turmas - de estudantes que ano a ano se sucedem.

Para que o ensino teórico possa ser ministrado com certa eficiência, dispõe atualmente a Secção de Micologia de cerca de 450 diapositivos sobre assuntos micológicos tais como culturas, formas de reprodução dos cogumelos, gráficos, aspectos diversos de micoses, etc.

Com o correr dos anos, tornou-se a secção de micologia um centro de pesquisas e dêsse modo numerosos problemas micológicos foram 
abordados. O primeiro estudo realisado foi sobre a blastomicose brasileira e teve como resultado esclarecer a etiologia dessa micose. Deixou de ser considerado como seu agente etiológico o Coccidioides immitis, passando a ser aceito o Paracoccidioides brasiliensis.

São hoje unânimes as opiniões dos cientistas estrangeiros e nacionais em considerar o Paracoccidioidè brasiliensis gérme da forma brasileira de blastomicose ou granuloma paracoccidióidico como diferente do Coccidioides immitis agente do granuloma coccidióidico, micose encontrada frequentemente nos Estados Unidos e algumas vêzes na Argentina.

O granuloma paracoccidióidico largamente distribuido no Brasil, particularmente no Estado de São Paulo constititue até certo ponto um problema social, quer pela sua disseminação quer por sua malignidade.

Até 30 de julho do corrente ano registramos 531 casos ocorridos no Brasil sendo dêstes 398 em São Paulo. Este número está longe de rep:esentar a verdade porque apenas se referem aos casos que foram publicados ou àqueles doentes que procuraram recurso nos grandes centros. Uma grande maioria de doentes, por falta de recursos financeiros ou por falta de assistência médica deve ter morrido no interior, pois que a quasi totalidade dos casos provém das zonas agrícolas. E a classe dos lavradores a que maiores tributos paga a tão mortífera entidade mórbida. Concorre ela com cerca de $80 \%$ do total dos casos.

As pesquisas por nós realisadas permitiram a pesquisadores estrangeiros assinalar a presença do granuloma paracoccidióidico fóra do Brasil. Assim, já foi êle assinalado uma dezena de vêzes na Argentina, 3 no Paraguai, outras tantas na Venezuela e uma vez no Uruguai, deixando assim de ser considerado como uma micose estritamente brasileira.

Sobre êsse tão importante problema estamos ha cerca de 3 anos realisando ensaios com uma vacina polivalente, preparada com mais de uma dezena de amostras de Paracoccidioides, escolhidas entre cêrca de uma centena de outras, isoladas de outros tantos casos clínicos. Os resultados obtidos com a vacinação têm sido brilhantes em muitos casos, mórmente quando associamos ao tratamento uma sulfanilamida.

Sobre a questão das blastomicoses em 'geral e de seus agentes, publicamos, sós ou em colaboração pouco mais de 40 trabalhos.

Além das blastomicoses, voltamos também nossas vistas para a questão dos micetomas. E' êste outro problema que merece ser melhor conhecido.

Do mesmo modo que para as outras micoses procuramos conhecer sua distribuição no Brasil: Conseguimos dados sobre 143 casos de micetomas sendo que 61 ocorridos em São Paulo. Êstes números também estão longe de ser a realidade, pelos mesmos motivos apontados acima.

Esta micose quando em início, pode facilmente ser tratada com a medicação iódica, porém depois que as lesões ganharam certa extensão e atingiram o tecido ósseo, necessário se torna uma intervenção cirúr- 
gica. Mas si a localisação do micetoma é cefálica, torácica ou visceral, a cirúrgia pouco ou nada pode fazer. Em casos de micetoma cérvico facial, ensaiamos com brilhantes resultados, uma vacina polivalente, preparada com a técnica aconselhada por Negroni, emérito pesquisador argentino.

A proposito desta localisação devemos dizer que já a encontramos duas vezes em estudantes de medicina, tendo sido o primeiro caso, a despeito do prognóstico reservado que apresentava, curado com a vacina e um tratamento associado de iodeto e raios X. O segundo iniciou ha pouco o tratamento.

Sobre micetomas publicamos, sós ou em colaboração, 12 trabalhos. A propósito dêste assunto devemos assinalar que por duas vêzes encontramos micetomas actinomicósticos com grãos negros, sendo que da segunda vez conseguimos isolar e estudar o cogumelo causador das lesões e dêsse estudo resultou a descrição de uma nova espécie do gênero Actinomyces e que denominamos paraguayensis, por ter o doente vindo do Chaco paraguaio, especialmente a São Paulo para se tratar.

Pelo que pudemos encontrar na literatura foi essa a primeira vez no mundo que se conseguiu isolar um Actinomyces produtor de grãos negros.

Um outro problema também interessante principalmente no ponto de vista etiológico é o da dermatite verrucosa ou cromomicose. A proposito dêste assunto realisamos em. 1936 com Morris Moore, micologista norte americano que veio à America do Sul estudar a blastomicose, um estudo micológico de numerosas amostras de cogumelos isolados de casos clínicos de cromomicose e chegámos a conclusão de que além dos gêneros descritos, vários outros pódem produzir a micose em apreço. Esta é ainda uma questão em aberto e sobre a qual muito se tem a fazer.

Os casos de cromomicose observados no Brasil sobem já a 59 sendo que sómente em São Paulo registramos 38. Os primeiros casos brasileiros de cromomicose assim como de granuloma paracoccidióidico foram observados em São Paulo, razão porque deve êsse estudo merecer de nossa parte uma especial atenção. Outro interessante problema micológico é o das micoses pulmonares. Em 1934 tivemos nossa atenção despertada para o assunto e iniciamos nossos estudos. De então para cá, várias vêzes publicamos os resultados de nossas pesquisas realisadas principalmente em colaboração com Carlos da Silva Lacaz, estudante monitor.

Desde aquela época até o momento realisamos mais de 500 exames de escarro para pesquisa de cogumelos. Os escarros enviados, geralmente tinham sido, uma ou mais vezes negativos para o agente etiológico da tuberculose. No entanto ainda em percentagem relativamente elevada foi êle por nós encontrado. Esses casos, suspeitos de uma micose pulmonar permitiram-nos isolar cogumelos em cerca de $35 \%$ dos escarros e a administração de iodetos aos pacientes foi geralmente seguida de acentuadas melhoras. Em poucos casos, uma vacina autógena muito contribuiu para a melhoria dos doentes. Infelizmente na 
maioria dos casos não nos foi possivel saber dos resultados da terapeutica aplicada, porque de regra recebiamos o material (escarro) para pesquisa de cogumelos e dos médicos assistentes poucas vêzzes tivemos noticias sobre a evolução do caso clínico.

Algumas vêzes, porém, pudemos acompanhar todo o evolver do processo e assim ter convicção de que efetivamente se tratava de uma micose pulmonar e não de uma tuberculose pulmonar conforme o diagnóstico clínico e radiológico.

Outros problemas sobre micoses tem sido tambem abordados pela secção de Micologia e longe iriamos só em sua enumeração. Sôbre assuntos micológicos foram já publicados por nós cerca de 110 trabalhos. Dentre êles devemos mencionar o livro "Micologia Médica", com 700 paginas e 300 figuras feito de acordo com a orientação dada ao curso de micologia, e destinado a proporcionar aos estudantes e aos médicos em geral, noções práticas sobre as micoses e seus agentes etiológicos. Sobre êsse livro, micologistas nacionais e estrangeiros, se manifestaram de modo muito honroso para a ciência micológica brasileira.

Quando ainda vigorava a exigência de tése para formatura, duas foram feitas na secção de micologia, uma sobre esporotricose e outra sobre os cogumelos do sapinho. Além dos trabalhos feitos em nossa colaboração, Carlos da Silva Lacaz, aluno monitor tem mais 5 publicações sobre assuntos micológicos.

Como pessoal auxiliar tem a secção de micologia, três técnicos encarregados da conservação da micoteca e seu arquivo, do preparo de meios de cultura e da parte de histologia.

Damos a seguir uma lista dos trabalhos realisados por nós sobre micologia.

\section{TRABALHOS DE MICOLOGIA DO DR. FLORIANO DE ALMEIDA}

1927

(1) Almeida (F.) \& Campos (E. de S.) - Contribuição para o estudo das "Blastomicoses" (Granul. cocc.) observados em São Paulo An. Fac. Med. Vol. II, 1927.

(2) Almeida (F.) \& Santos (L.) - Sobre um caso de Blastomicose pulmonar - An. Fac. Med. Vol. II, 1927.

\section{8}

(3) Almeida (F.) - Lesões cutaneas da Blastomicose em cobaros experimentalmente injetados - An. Fac. Med. Vol. III, 1928.

(4) Almeida (F.) - Sobre a localisação cutanea da blastomicose em um cobaio inoculado experimentalmente no testículo - Ciência Médica, ano IV, n. ${ }^{\circ} 4,1928$.

\section{9}

(5) Almeida (F.) - Aspectos histológicos dos casos de blastomicoses verificados em São Paulo - Brasil Médico, 4-5-1929. Com. à Semana Dermatológica. Soc. Med. de São Paulo, 1929.

(6) Almeida (F.) - Notas sobre a piedra brasileira - Rev. de Mod.. ano XIII, n. ${ }^{\circ}$ 51, 1929.

(7) Almeida (F.) - Incidência da blastomicose no Brasil - Bol. Biol., Fasc. XV, 30-6-1929. 
(8) Almeida (F.) - Blastomicose experimental - Bol. Biol., Fasc. XV, 6-1929.

(9) Almeida (F.) - Estudo comparativo do granuloma coccidióidico nos Estados Unidos e Brasil - An. Fac. Med., Vol. IV, 1929.

(10) Almeida (F.) - Estudo sobre o parasito do granuloma coccidióidico Bol. Biol., Fasc. 16, 20-12-1929.

\section{0}

(11) Almeida (F.) + Notas sobre o estudo das tinhas - Rev. Med., ano XIV, n.o 52, 1930.

(12) Almeida (F.) - Micetoma actinomicósico de grãos pretos - Com. à Soc. Biol. de São Paulo.

(13) Almida (F.) - Cromoblastomicose em São Paulo - Com. à Soc. Biol. de São Paulo.

(14) Almeida (F.) - Diferença entre o agente etiológico do granuloma coccidıóidico nos Estados Unidos e no Brasil. Um novo gênero para o parasito brasileiro - Com. à Soc. Biol. de São Paulo, 10-9-1930. Rev. Biol. Hig., Vol. II, Fasc. III - IV, 1931.

(15) Almeida (F.) - Différences entre l'agent étiologique du granulome coccidioidique des Etats Unis et du Brésil. Un novau genre pour le champignon brésilien - Compt. Rend. Soc. Biol. TCV, n. ${ }^{\circ} 2$, pg. 315, 1930.

(16) Almeina (F.) - Em torno do problema da blastomicose brasileira Conf. Soc. Med. de São Paulo, $1-12-1930$.

(17) Almeida (F.) - Micetoma actinomicósico de grãos pretos - An. Fac. Med., Vol. V, 1930.

(18) Almeida (F.) - Estudo comparativo do granuloma coccidióidico no Brasil e noś Estados Unidos. Novo genero para o parasito brasile1ro, - An. Fac. Med., Vol. V, 1930.

\section{1}

(19) Almeida (F.) - Granuloma paracoccidióidico. (Trabalho para concorrer a uma vaga na Soc. de Med. de São Paulo) - Bol. Soc. Med., Vol. XV, ns. 4, 5, 6 - Junho-Julho-Agosto de 1931.

(20) Almeida (F.) \& Santos (L.) - Peritonite e perfuração do estomago por Paracoccidioides brasiliensis - Com. à Sem. Biol., 24-9-31.

(21) Almeida (F.) \& Maciel (J.) - Aspergilose pulmonar aviaria Com. à Sem. Biol., 24-9-31.

(22) Almerda (F.) - Granuloma coccidióidico experimental - Com. à Soc. Biol., 8-12-931.

1932

(23) Almeida (F.) - Epidemiologia da blastomicose brasileira (Gran. parac. no Brasil - Com. à Soc. Med. de São Paulo. Janiero de 1932.

Almeida (F.) - Considerações em torno do Coccidioides immitis Com. à Sem. Soc. Med. de São Paulo, '1932.

Almeida (F.) \& Santos (L.) - Peritonite e perfuração do estomago por Blastomicose - Com. à Sem. Lab. Soc. Med. Cir. de São Paulo, 1932.

Almeina (F.) - Considérations sur les genres Coccidioides immitis et Pseudococcidioides Mazzai - Compt. Ren. Soc. Biol. de Parasitol., 1932.

Allmeida (F.) - Considerações em torno do Coccidioides immitis e Psendococcidioides Mazzai - An. Fac. Med. S. Paulo, 1932, Vol. I, Parte II.

Almeida (F.) - As blastomicoses no Brasil. (Trabalho premiado pela Ac. Nac. Med. Rio de Janeiro. Premio oficial. Medalha de

ouro). An. Fac. Med. São Paulo V. IX. 1932.
Almeida (F.) - Contribuição para o estudo da morfologia do Coccidioides immitis nos tecidos parasitados. 
(30) Almeida (F.) - Considerações sobre o diagnóstico histopatologico dos micetomas - Rev. Biol., Vol. II. Dezembro de 1932.

1933

(31) Almeida (F.) - Notas sobre a morfologia do $P$. brasiliensis nos tecidos parasitados - Com. à Soc. Biol., '1933.

(32) Almeida (F.) - Nota a proposito do Coccidioidis immitis e Pseudococcidioides Mazzai - Com. à Soc. Biol., em 9-11-1933. An. Fac. Med., Vol. 10, Fasc. 1. 1934.

(33) Almeida (F.) - Blastomicose em geral e sua classificação - Rev. Paulista de Med., Vol. 3, n. ${ }^{\circ}$ 5, Novembro de 1933.

(34) Almieida (F.). \& Camargo (Inah) - Agente etiológico do sapinho. Estomatite cremosa ou pseudomembranosa - Rev. Biol. Hig., Vol. 4, n. ${ }^{\circ}$ 3, Dezembro de 1933.

(35) Almejdd (F.) - Influencia da temperatura no aspecto das culturas do P. brasiliensis - Com. à Soc. Biol., 1933.

1934

(36) Almeida (F.) - Formações radiadas da membrana dos cogumelos parasitos. - Com. à Soc. Med. Cir. de São Paulo, Maio de 1933 An. Fac. Med. de São Paulo, Vol. 10, Fasc. 2, ano de 1934.

(37) Almeida (F.) - Considerações sobre micoses pulmonares - Com. à Soc. Med. de São Paulo, sessão de 1-12-1933.

(38) Almèrd (F.) - Le Blastomicosi nel Brasile - Folia Clinica et Biologica. Ano V, Vol. 5, 1934.

(39) Almeida (F.) - Ligeiras notas sobre micoses - Com. à Soc. Biol. de São Paulo, janeiro de 1934.

(40) Almeida (F.) - Considerações em torno de um cogumelo encontrado por Carini nos pulmões dos sapos: "Lepdodactylus pentadactylus". Com. à Soc. Biol. de São Paulo, Março 1934.

(41) Almeida (F.) - A proposito de um caso de blastomicose por P. brasiliensis - ocorrido na Argentina - Com. à Soc. Biol., S. Paulo, 3-934.

(42) Almeida (F.) - Asma provocada por cogumelos. (Nota prévia) Com. à Soc. Biol., S. Paulo, 3-934.

(43) Almetda (F.) - Duplicidade de Actinomyces em lesões actinomicosicas - Com. à Soc. Biol., S. Paulo, 5-934.

(44) Allmeida (F.) - Nova contribuição para o estudo morfológico no C. immitis nos tecıdos parasitados - Com. à Soc. Biol. S. Paulo, 6-934.

(45) Almeida (F.) - Cromoblastomicose experimental em sapos - Com. à Soc. Biol. S. Paulo, 6-934.

(46) Almeida (F.) - Notas sobre uma denominação genérica - Com. à Soc. Biol. S. Paulo, 7-934.

(47) Almeida (F.) - Contríbuição para o estudo dos agentes etiológicos das blastomicoses brasileiras - Com. à Soc. Med. Cir. do Rio de Janeiro.

(48) Almeipa (F.) - Contribuição para o estudo cultural dos cogumelos: C. immitis, $P$. brasiliensis e Gilchristia dermatitidis - Com. à Soc. Biol. S. Paulo, 2-11-934.

(49) Almeida (F.) - Considerações em torno dos agentes etiologicos das blastomicoses - An. Paulistas de Medicina, Vol. II, 1935.

(50) Almeida (F.) - Breves considerações em torno dos micetomas, Arquivos Biologicos, Janeiro e Fevereiro de 1935.

(51) Almeida (F.) - Os micotorulados como causadores de micoses Com. à Soc. Biol. S. Paulo, 8-2-935.

(52) Almeina (F.) - Dualidade de Actinomyces em torno de um caso actinomicose - An. Fac. Med. S. Paulo, Vol. II, 1935. 
(53) Armeida (F.) - Ação do mel sobre as culturas do $P$. brasiliensis Com. à Soc. Biol. S. Paulo, 8-3-935.

(54) Almeida (F.) - Tinhas. Seu tratamento - Arquivo de Biologia, n. ${ }^{c}$ 187, ano 19, 1935.

(55) Almeida (F.) - Micoses do aparelho respiratorio - Conf. na Soc. Med. S. Paulo, 16-8-1935.

(56) Almeida (F.) \& MORRIs MOORE - Agentes etiológicos da cromomicose. (cromoblastomicose de 'Terra, Torres, Fonseca \& Leão, 1923) Nas Américas - Com. à Soc. Biol., 13-12-1935.

\section{6}

(57) Almeida (F.) - Considerações sobre a inoculação cardiaca do $C$. immitis e do $P$. brasiliensis - Folia Clinica et Biologica, ano VII, n.o 3, 1936.

(58) Alméida (F.) \& Moore (Morris). - Novo gênero e nova espécie de fungo produtor de cromomicose. (Phialoconidiophora Guggenheimia) - Com. à Soc. Biol., 8-1-1936.

(59) Almeida (F.) \& MOORE (Morris) - Piedra por Trichosporum Com. à Soc. Biol., 8-1-1936.

(60) Armeida (F.) - Granuloma paracoccidióidico e sua localișação bucal - Rev. Otolaryngológica de São Paulo, Vol. IV, .1936, n..$^{\circ} 5$.

(61) Almeida (F.) - Cromomicose - Arquivo de Biologia, 1936.

(62) Almeida (F.) - Granuloma paracoccidióidıco: sua distribuição no Brasil e particularmente em São Paulo - An. Fac. Med., Vol. XII, 1936.

(63) Almeida (F.) - Considerações em torno da distribuição dos micetomas no Brasil - Folia Clinica et Biologica, Vol. VII, n. ${ }^{\circ}$ 5, 1936.

AlmeIDA F.) \& Moore (Morris) - New organisms of chromomycosis - Anals of Missouri Botanical Garden, 23:543-522 - Nov. 1936.

1937

(65) Almeida (F.) - Micose pulmonar aviaria por Aspergillus - Folia Clinica et Biologica, n. ${ }^{\circ} 3,1937$.

(66) Almeida (F.) \& LaCAZ (C. da S.) - Observações em torno da espécie "Candida butantanensis" de J. M. Gomes - Folıa Clinica et Biologica, n. ${ }^{\circ} 1$.

\section{8}

(67) Almeida (F.) - Observações sobre culturas de $P$. cerebriformes de Moore, 1935 - An. Fac. Med., Vol. XIV, pag. 235, 1938.

(68) Almeida (F.) - Considerações em torno das epidermomicoses geralmente conhecidas como manifestações de ácido úrico - Arquivo de Biol., ano XXII, n. ${ }^{\circ} 207$, Julho de 1938.

(69) Almeida (F.) \& AbDalla (Rosa) - Nota a propósito de alguns cogumelos do gênero Torulopsis - An. Fac. Med., Vol. XIV, pag. 213, 1938.

(70) Almeida (F.) \& Lacaz (C. da S.) - Considerações em torno do sapinho vaginal e bucal - Rev. Ginecologia, Vol. III, Fasc. I, 1938.

(71) Almeida (F.) - Considerações sobre micoses pulmonares - Conf. na Soc. Tisiol., 23-5-1938.

(72) Almeida (F.) - Técnica micológica (Cap. do Livro) - Rev. Biol. ano XXII n. ${ }^{\circ} 211-1938$.

(73) Almeida (F.) - Vacina contra o granuloma paracoccidióidico Folia Clinica et Biologica. Vol. X, n.0 6, 1938.

\section{9}

(74) Almeida (F.) - Micologia Médica - Companhia de Melhoramentos de São Paulo, 1939.

(75) Almeida (F.) \& Lacaz (C. dá S.) - Considerações sobre um caso de glossite superficial membranosa de natureza micótica - Folia Clinica et Biologica, Vol. XI, n. ${ }^{\circ} 1,1939$. 
(76) Almeida (F.) \& LACAZ (C. da S. - Breves considerações sobre tuberculose ganglionar e blastomicose - Rev. Biol. e Hig., 9(2)101-103, Dezembro de 1938.

(77) Almeida (F.) \& LaCAZ (C. da S.) - Estudos micológicos sobre tricomicose nodular - Rev. Biol. Hig. 9(2)101-103, Dezembro de 1938.

(78) Almeida (F.) \& Abdalla (Rosa) - Micetomas torácicos — Án. Fac. Med. S. Pauko, XV. 1939.

(79) Almeida (F.) \& LacAz (C. da S.) - Frequencia das micoses pulmonares em S. Paulo - Rev. Med., Vol. 23 - Junho 1939 - n. ${ }^{\circ} 66,9$ - Pag. 33-43.

(80) Almeida (F.) \& Lacaz (C. da S.) - Primeiro caso de acromia parasitária de Jeanselme em São Paulo - An. Fac. Med., XV - 1939.

(81) Almeida (F.) - Aspergillus do grupo ochraceus isolado de escarro An. Fac. Med., 1939. XV - pag. 141.

(82) Almeida (F.) - Estudo micológico de um Actinomyces de grão pretos - Micopatologia, Vol. II, 940 - Fasc. 3, pag. 201.

(83) Almeida (F.) \& LACAZ (C. da S.) - Considerações em torno das micoses cirurgicas - Arquivos de Cirurgia. Clin. Exptr., Vol. III, n. ${ }^{\circ}$ 2, Abril de 1939.

(84) Almeida (F.) - Micoses cirúrgicas - Semana de Cirurgia, Julho de 1939 - Boletim do Sanatório S. Lucas - Vol. I, Nov. 1939, n. 5 e 4.

(85) Almeida (F.) \& LaCAZ (C. da S.) - Cogumelò do gênero Histoplasma isolado de lesões de cromomicose. Associações de fungos nas lesões - Folia Clinica et Biologica, Vol. XI, n. 3, 1939.

(86) Almeida (F.), Lacaz (C. da S.) \& Fragale - Micose pulmonar por cogumelo do gênero Rhyzopus. (Considerações sobre um caso) Therapia, ano I, n. 4, julho e agosto de 1939, S. Paulo.

(87) Almeina (F.) LaCAZ (C. da S.) - Otomicose por cogumelo do gênero Aspergilus - Imprensa Médica, 1939.

(88) A.MeIdA (F.) - Actinomicose de grãos pretos - Folia Clinica et Biologica, Vol. XII, n. 1, 1940.

(89) Almeida (F.) \& Lacaz (C. da S.) - Consıderações em torno de um caso de blastomicose cutâneo-mụcosa - An. Paul. Med. Cir., Vol. XXXVIII - Out. 1939, n. 4.

(90) Almeida (F.) - Importancia prática das micoses - Palestra na Soc. Médica de S. Lucas, 12-7-939 — Bol. do San. S. Lucas, Vol. I, n. 9, Março de 1940.

(91) Almeida (F.) \& Barbosa (S.) - Sobre uma Cunninghamela elegans isolada de um inseto - Folia Clinica et Biologica.

(92) Almeida (F.) - Cogumelos produtores de doenças - Viver, n. 17, ano 2, 1939.

(93) Almeida (F.) - Cogumelos uteis ao homem - Viver, n. 18, ano 2, 1939.

1940

(94) Almeida (F.) \& Lacaz (C. da S.) - Actinomicose cérvico-facial Arquivos de Cir. Cl. e Exp. (em publicação).

(95) Almeida (F.) \& Barbosa (S.) - Contribuição parà o estudo dos micetomas maduromicóticos observados no Brasil - An. Fac. Med., Vol. XVI, 1940.

(96) Almeida (F.) \& Lacaz (C. da S.) - Sobre um caso de granuloma paracoccidióidico com curiosos aspectos morfológicos do parasito no tecido - Folia Clinica et Biologica, Vol. XII, n. 1, 1940.

(97) Almeida (F.) \& Barbosa (S.) - Contribuição para o estudo micológico do Cephalosporium recifei - em publicação.

(98) Almeida (F.) \& Lacaz (C. da S.) - Cogumelo do gênero Geotrichum isolado de lesões do reto -Folia Clinica et Biologica, Vol. XII. n. 2, 1940. 
(99) Almeida (F.) \& LACAz (C. da S.) - Considerações micológı́cas sobre 4 amostras do gênero Geotrichum - Folia Clinica et Biologica, Vol. XII, in. 2, 1940.

(100) Almeida (F.) \& Lacaz (C. da S.) - Processos pulmonares mixtos. Com especial referencia à associação tuberculo-micótica. Anais Paul. Med. e Cir. V. XXIX, Maio 1940, n. ${ }^{\circ} 5$.

(101) Almeida (F.) \& Lacaz (C. da S.) - Cogumelo do genêro Saccharomyces isolado de escarro - An. Fac. Med. Vol. XVI, 1940.

(102) Almeida (F.) \& LacAz (C. da S.) - Estudo micológico de um cogumelo do gênero Hormodendrum isolado de acromı parasitária - An. Fac. Med., Vol. XVI, 1940.

(103) Almeida (F.) \& LAcaZ (C. da S.) - Nova técnica para a demonstração de ascosporos - Folia Clinica et Biologica. V.XII, n. ${ }^{\circ}$ 4, 1940.

(104) Almeida (F.) \& LACAz (C. da S.) - Considerações micológicas sobre 6 amostras de lêvedos isoladas de esçarro - An. Fac. Med., Vol. XVI, 1940.

(105) Almeida (F.) \& Lacaz (C. da S.) - Contribuição para o estudo de actinomicose de grãos vermelhos - An. Fac. Med. Vol. XVI, 1940.

(106) Almeida (F.) - Estado atual da questão das cromomicoses - Rev. Med., Vol. XXIV, Junho de 1940, n. 78.

(107) Almeida (F.) \& LACAz (C. da S.) - Cogumelos levediformes isolados de bile - Folia Clin. et Biol. n. 3, Vol. XII, 1940.

(108) Almeida (F.) - Cogumelos provocadores de lesões da pele conhecidas Como ácido úrico - Viver, n. 25, Julho de 1940.

(109) Almeida (F.) \& Abdalla (Rosa) - Estudo micológico de algumas amostras do gênero Actinomyces $\rightarrow$ An. Fac. Med., Vol. XII, 1940.

(110) Almeida (F.) - Lesões cutâneas produzidas por cogumelos - Viver, n. 28, 1940, outubro.

(111) Almeida (F.) - A secção de Micologia do Departamento de Microbiologia e Imunologia - Rev. Med., Vol. XIV, n. 81, setembro, 1940.

\section{TRABALHOS DE CARLOS DA SILVA LACAZ (monitor acađêmico)}

1 - Cultura do escarro para pesquiza de cogumelos. Fungos productores de mycos bronquio-pulmonares (Brasil Medico, anno LIII - n. 13 e 14 - 25 de março e $10^{\circ}$ de abril de 1939 - Rio de Janeiro).

2 - Pneumonycoses - Revista Clínica de São Paulo - Vol. IV; n. 1, julho de 1938.

3 - Chromomycoses - Brasil Médico - n. 24 e 25 - 11 e 18 de junho de 1938, ano LII.

4 - Tinhas da pele e dos pêlos - Imprensa Médica - ano XIV, n. 266, 1. de julho de 1938.

5 - O iodo no tratamento das micosees - Anais Paulistas de Medicina e Cirurgia - Vol. XXXIX, maio de 1940, n. 5.

\section{TES ES}

GrandinetTI, L. - Contribuição para o estudo da esporotricose em S. Paulo CAmargo, I Fac. Med. São Paulo - 1934. Paulo - Fac. Med. São Paulo - 1934.

Em 1939 o Dr. Frederico Simões Barbosa, fez na secção de Micologia, um estágio e além dos trabalhos em colaboração, realisou outros. Um dêles, Concerning a new hyphomycete parasite of man: Cephalosporium cordoniformis n. sp., foi publicado no fasc. IV ano II de Mycopathologia, 1940.

Além disso elaborou uma tese a ser defendida brevemente. 